\title{
A Delicately Designed Sulfide Graphdiyne Compatible Cathode for High-Performance Lithium/Magnesium-Sulfur Batteries
}

\author{
Huiping Du, Zhonghua Zhang, Jianjiang He, Zili Cui, Jingchao Chai, Jun Ma, Ze Yang, \\ Changshui Huang,* and Guanglei Cui**
}

\begin{abstract}
Novel sulfur cathodes hold the key to the development of metal-sulfur batteries, the promising candidate of next-generation high-energy-storage systems. Herein, a fascinating sulfur cathode based on sulfide graphdiyne (SCDY) is designed with a unique structure, which is composed of a conducting carbon skeleton with high $\mathrm{Li}^{+}$mobility and short sulfur energy-storing unites. The SCDY cathode can essentially avoid polysulfide dissolution and be compatible with commercially available carbonate-based electrolytes and Grignard reagentbased electrolytes (all phenyl complex (APC) type electrolytes). Both the assembled Li-S and Mg-S batteries exhibit excellent electrochemical performances including large capacity, superior rate capability, high capacity retention, and high Coulombic efficiency. More importantly, this is the first implementation case of a reliable $\mathrm{Mg}-\mathrm{S}$ system based on nucleophilic APC electrolytes.
\end{abstract}

dissolved in carbonate-based solvents, leading to poor cell reversibility and fast capacity fading. Glyme-based and dioxolane solvents can dissolve the high-order polysulfides easily, yet accompanied by the high-order polysulfides migrating between the cathode and anode, leading to the socalled "shuttle effect," which will result in the loss of active materials and low Coulombic efficiency. ${ }^{[8]}$

Many efforts have been made to design sulfur cathode structures especially for $\mathrm{Li}-\mathrm{S}$ batteries. Various carbon materials with excellent conductivity, large surface area, and strong adsorption ability have been used to accommodate S. ${ }^{[1,9-11]}$ However, those sulfur cathodes were generally

\section{Introduction}

Metal-sulfur batteries are promising to be next-generation high-energy-storage systems due to high capacities of both the sulfur cathode and metal anode. ${ }^{[1,2]}$ Sulfur cathode has a high theoretical specific capacity of $1672 \mathrm{mAh} \mathrm{g}^{-1}$ and theoretical volumetric capacity of $3459 \mathrm{mAh} \mathrm{cm}^{-3}$, much higher than conventional lithium-ion battery cathodes such as $\mathrm{LiCoO}_{2}$ and $\mathrm{LiFePO}_{4} \cdot{ }^{[3]}$ It should also be noted that as one of the most abundant element resources in the earth, sulfur is inexpensive, which is favorable for grid scale storage systems..$^{[4-6]}$

However, the electronic and ionic conductivities of sulfur are both low, which will give rise to poor electrochemical accessibility and thus low utilization. ${ }^{[7,8]}$ Besides, sulfur (cyclo- $\mathrm{S}_{8}$ ) discharges stepwise, involving two-step processes: $\mathrm{S}_{8}$ is reduced to high-order polysulfides and then reduced to low-order polysulfides. The high-order polysulfides can be partially

Dr. H. P. Du, Dr. Z. H. Zhang, Dr. J. J. He, Dr. Z. L. Cui, Dr. J. C. Chai, Dr. J. Ma, Dr. Z. Yang, Prof. C. S. Huang, Prof. G. L. Cui Qingdao Industrial Energy Storage Technology Institute Qingdao Institute of Bioenergy and Bioprocess Technology Chinese Academy of Sciences

Qingdao 266101, P. R. China

E-mail: huangcs@qibebt.ac.cn; cuigl@qibebt.ac.cn

Dr. H. P. Du, Dr. Z. H. Zhang, Dr. J. J. He, Dr. J. C. Chai

University of Chinese Academy of Sciences

No. 19A Yuquan Road, Beijing 100049, China

DOI: 10.1002/smll.201702277 obtained by heating sulfur with carbon matrix and sulfur mainly existed as $\mathrm{S}_{8}$; thus shuttle effect was only partially restrained because liquid electrolytes could also access sulfur that physically diffused in carbon matrix. ${ }^{[1]}$ Recently, Guo and co-workers demonstrated that the shuttle effect was avoided essentially as sulfur exists as small molecular forms $\mathrm{S}_{2-4}$ by confining sulfur into the micropores $(0.5 \mathrm{~nm}) \cdot{ }^{[12]}$ Subsequently, Wang and coworkers synthesized $\mathrm{S}_{2}$-intercalated reduced graphite oxide compound via in situ $\mathrm{S}$ reduction and intercalation, which shows high and stable capacities. ${ }^{[13]}$ These two studies open the door for designing novel sulfur cathodes based on small sulfur mode.

Graphdiyne (GDY), as a new carbon allotrope, possesses many interesting properties due to its unique structure. It is a kind of planar and layered material. Compared to graphene, the introduction of butadiyne linkages $(-\mathrm{C} \equiv \mathrm{C}-\mathrm{C} \equiv \mathrm{C}-)$ to benzene rings leads to lower atom density and gives rise to natural holes. First-principle calculations reveal that the unique atomic arrangement and electronic structures of GDY facilitate both inplane and out-plane diffusion of $\mathrm{Li}$ ions with moderate barriers of $0.53-0.57 \mathrm{eV}$, making GDY with high $\mathrm{Li}^{+}$mobility. ${ }^{[14]}$ Our previous works have also reported GDY to be a promising anode of lithium-ion batteries and lithium-ion capacitors, confirming that GDY is a conductive carbon skeleton with high $\mathrm{Li}^{+}$mobility. ${ }^{[15-17]}$

We here designed a fascinating sulfur cathode by taking structure advantage of GDY. GDY, with uniformly distributed pores $(5.42 \AA)$ and large interlayer distance $(0.365 \mathrm{~nm})$, would be a good reservoir for short sulfur molecules $\left(S_{2-4}\right) \cdot{ }^{[18,19]}$ The highly reactive carbon-carbon triple bonds in GDY would easily react with sulfur radicals. In addition, GDY is a conducting 
carbon skeleton with high Li-ion mobility (Li-ion diffusion both in-plane and out-plane). By using a simple thermal synthesis procedure, short sulfur unites generated through the cleavage of $\mathrm{S}_{8}$ rings would react with the highly reactive carbon-carbon triple bonds of GDY and confined in the triangle-like pores inside GDY. As a result, short sulfur energy-storing unites were entrapped in a conductive carbon skeleton with high $\mathrm{Li}^{+}$ mobility through both covalent bonding and physical confinement. The structure of the sulfur cathode based on sulfide graphdiyne (SGDY) was characterized by Raman, IR, X-ray photoelectron spectroscopy (XPS), thermogravimetric analysis (TGA), and so on. Finally, we applied the SGDY cathode to Li-S batteries using commercially available carbonate-based electrolytes and Mg-S batteries using all phenyl complex (APC) type electrolytes, both of which show excellent electrochemical performances. Our delicately designed strategy could open a new avenue for preparing sulfur cathode from molecular design.

\section{Results and Discussion}

\subsection{Structure Characterization}

SGDY was prepared by a simple thermal synthesis procedure as shown in Figure 1a. At $350^{\circ} \mathrm{C}, \mathrm{S}_{8}$ rings were split into short chains $\left(\mathrm{S}_{x}, 1 \leq x \leq 4\right)$ and anchored in the triangular pores of GDY by chemically bonding to carbon-carbon triple bonds. ${ }^{[20,21]}$ While the residual $S_{x}$ recombined into $S_{8}$ rings when the temperature cooled down to the room temperature, which was removable by $\mathrm{CS}_{2}$ washing.

The morphologies of pristine GDY and SGDY are presented in Figure 1b-g. The pristine GDY powder (Figure 1b) is composed of connected nanoparticles with rough surface; the typical transmission electron microscope (TEM) image (Figure 1c) demonstrates pristine GDY has layered structure and the layer space is $0.365 \mathrm{~nm}$ as shown in Figure 1d. Compared with GDY, the morphology of SGDY does not change as indicated in Figure 1e-f. SGDY also has layer structure while the high-resolution (HR) TEM image of SGDY shows that SGDY becomes amorphous (Figure 1g). The elemental mapping images of $\mathrm{C}$ and $\mathrm{S}$ clearly show the uniform distribution of the carbon and sulfur atoms in SGDY and the S content is $26.11 \mathrm{wt} \%$.

Raman spectroscopy is a powerful tool to characterize carbon materials. ${ }^{[22,23]}$ Figure 2a shows the Raman spectra of pristine GDY and SGDY, both of which exhibit two prominent peaks: $\mathrm{D}$ band and $\mathrm{G}$ band. New peaks in Figure $2 \mathrm{~b}$ can be assigned to $\mathrm{C}-\mathrm{S}$ bonds and $\mathrm{S}-\mathrm{S}$ bonds formed in SGDY. Furthermore, the peak of GDY at $2173.7 \mathrm{~cm}^{-1}$ attributed to the vibration of the diacetylenic linkages $(-\mathrm{C} \equiv \mathrm{C}-\mathrm{C} \equiv \mathrm{C}-$ ) disappears for SGDY, indicating carbon-carbon triple bonds of GDY react with sulfur
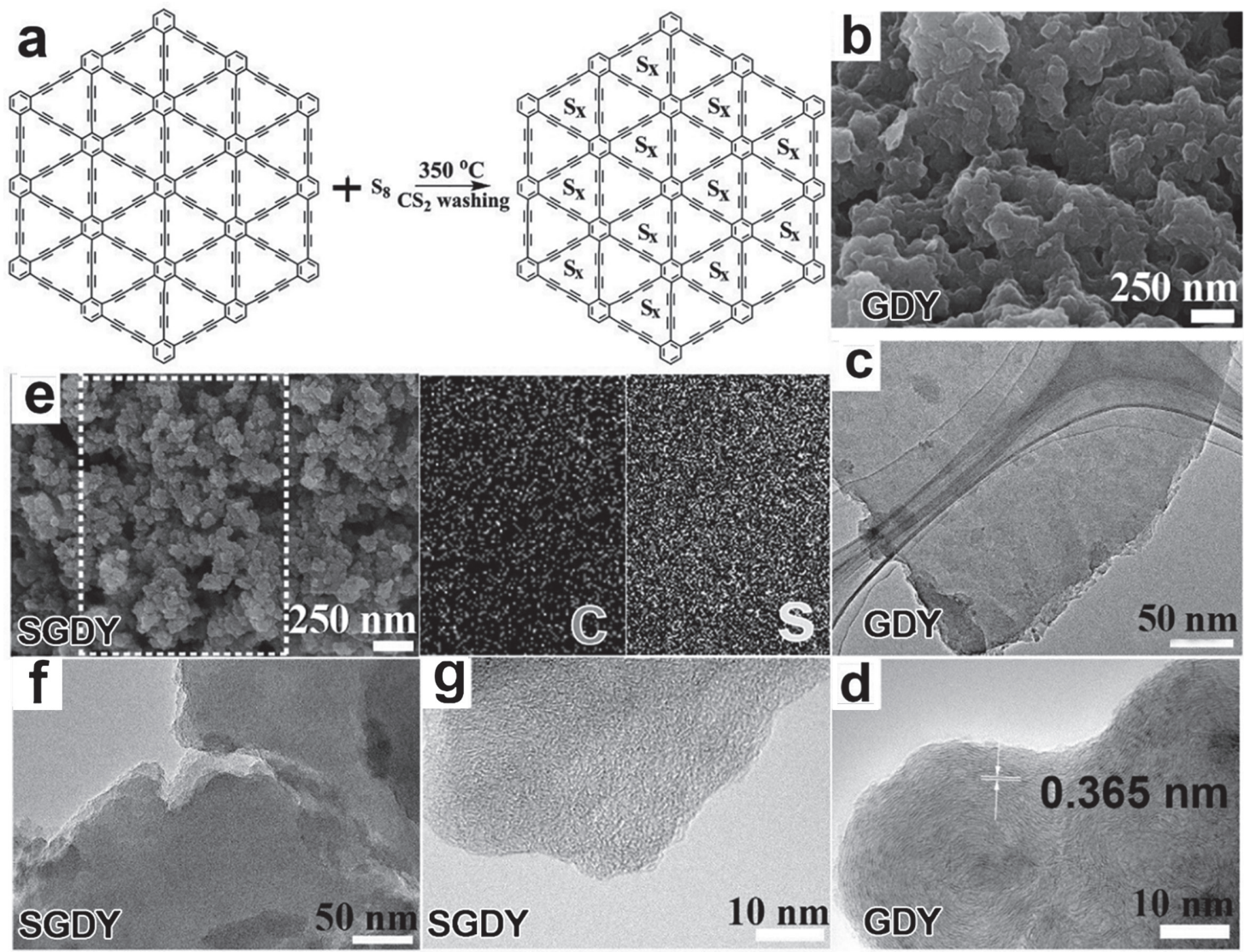

Figure 1. a) Schematic illustration of the preparation process of SGDY by using a simple thermal synthesis procedure. b) SEM image of GDY powder. c) TEM image of GDY powder. d) HRTEM image of GDY powder. e) SEM image and corresponding element mapping in the dashed box of SGDY. f) TEM image of SGDY. g) HRTEM image of SGDY. 

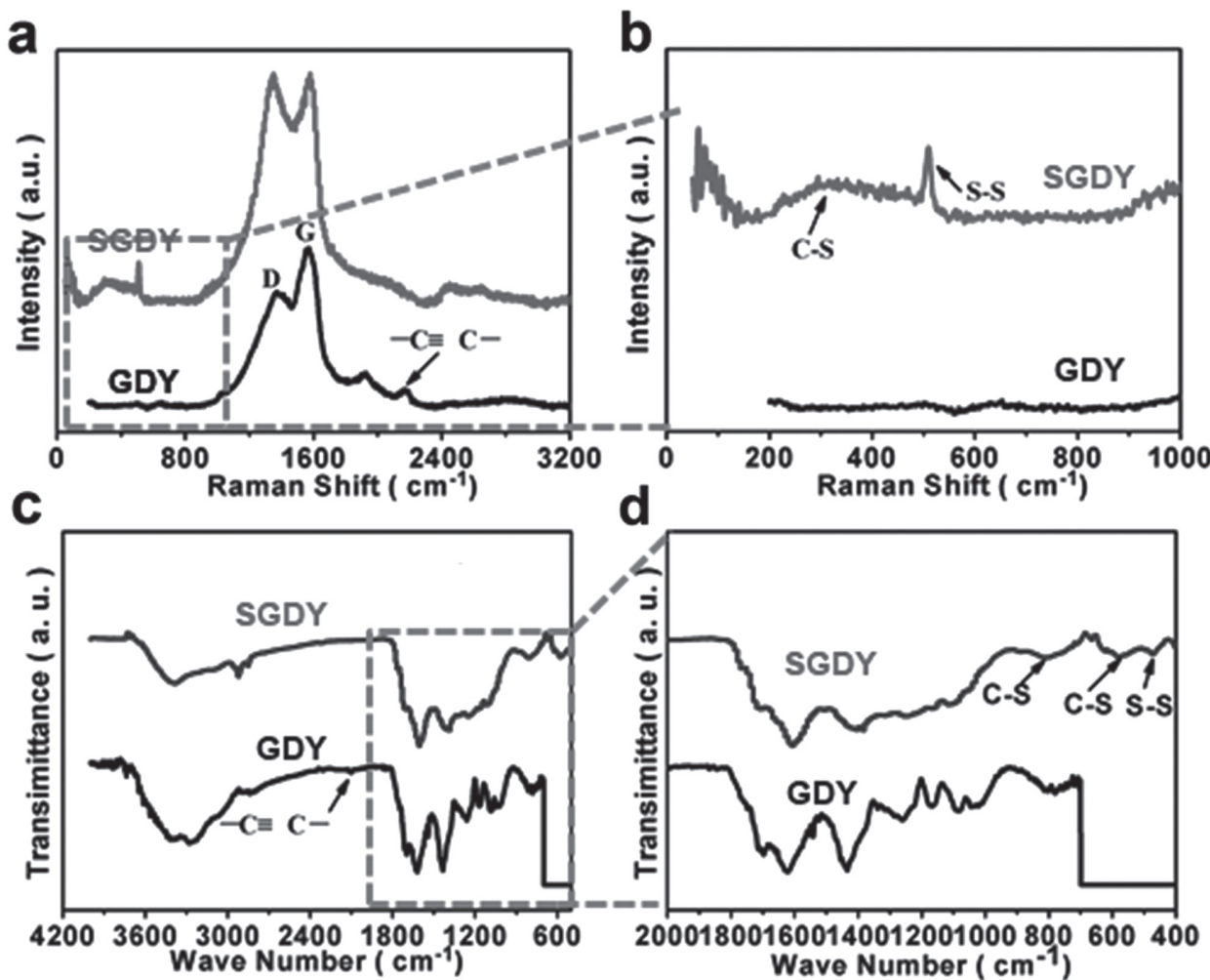

Figure 2. Raman spectra of a) GDY and SGDY and b) selective enlargement. FTIR spectra of c) GDY and SGDY and d) selective enlargement.

atoms to generate $\mathrm{C}-\mathrm{S}$ bonds and $\mathrm{S}-\mathrm{S}$ bonds. ${ }^{[20,24,25]}$ In addition, the ratio of the intensities of the $\mathrm{D}$ and $\mathrm{G}$-bands $\left(I_{\mathrm{D}} / I_{\mathrm{G}}\right)$ of SGDY is 1 , which is larger than that of the pristine GDY $(0.62)$, indicating more defects caused by $\mathrm{S}$ introduction. The Fouriertransform infrared spectroscopy (FTIR) spectra of SGDY further confirm the presence of $\mathrm{C}-\mathrm{S}$ bonds $\left(671\right.$ and $804 \mathrm{~cm}^{-1}$ ) and $\mathrm{S}-\mathrm{S}$ bonds $\left(513 \mathrm{~cm}^{-1}\right)$ compared to pristine GDY, as shown in Figure 2c,d. ${ }^{[20,24,26]}$

XPS test was also employed to confirm the generation of SGDY structure. As shown in Figure 3a, GDY is a kind of allcarbon material; the signal for $\mathrm{O} 1 \mathrm{~s}$ orbitals in the XPS survey spectrum of GDY arises from the adsorption of $\mathrm{O}_{2}$ in air before XPS test. A new peak at $163.7 \mathrm{eV}$ assigned to $\mathrm{S} 2 \mathrm{p}$ peak appears in the XPS survey spectrum of SGDY, indicating the existence of sulfur element (Figure 3b). Figure 3c shows the high-resolution C 1s XPS spectra of GDY and SGDY. The area ratio of the $\mathrm{sp}(\mathrm{C} \equiv \mathrm{C}, 285.1 \mathrm{eV})$ and $\mathrm{sp}^{2}(\mathrm{C}=\mathrm{C}, 284.6 \mathrm{eV})$ hybridized carbon atoms is 2, confirming GDY features benzene rings linked through diacetylenic linkages. The bonding between carbon and oxygen such as $\mathrm{C}=\mathrm{O}$ bonds may be ascribed to the chemical adsorption of oxygen on the surface of GDY or the reaction between oxygen and some terminated acetylenic bond exposed on the surface of GDY, similar to the situation reported in previous references. ${ }^{[15-17]}$ In contrast, C 1s XPS spectra of SGDY have an additional peak at $286.1 \mathrm{eV}$, which can be assigned to C-S bonds. ${ }^{[27,28]} \mathrm{S} 2 \mathrm{p}$ peak (Figure $3 \mathrm{~d}$ ) is split into two peaks at 163.5 and $164.5 \mathrm{eV}$, which can be ascribed to the $\mathrm{S} 2 \mathrm{p} 3 / 2$ and $\mathrm{S} 2 \mathrm{p} 1 / 2$, respectively. That the binding energy of the $S 2 \mathrm{p} 3 / 2$ peak $(163.5 \mathrm{eV})$ is lower than that of $\mathrm{S}_{8}(164.0 \mathrm{eV})$ indicates the presence of $\mathrm{C}-\mathrm{S}$ bonds instead of $\mathrm{S}_{8} \cdot{ }^{[27,29,30]}$ Moreover, in the $\mathrm{C}$ 1s XPS spectra of SGDY, the area ratio of the sp and $\mathrm{sp}^{2}$-hybridized carbon decreases compared to GDY, indicating sulfur is linked to the $\mathrm{C} \equiv \mathrm{C}$ bonds instead of benzene rings in GDY. Meantime, it is known that the triangle-like pore size and layer distance of GDY are $\approx 0.5 \mathrm{~nm}$ and $0.365 \mathrm{~nm}$, respectively. ${ }^{[18,19]}$ Combined with the size of sulfur molecular chains, only shortsulfide unites can be accommodated in the carbon skeleton of GDY as only the dimension of $\mathrm{S}_{2-4}$ is $<0.5 \mathrm{~nm}$. ${ }^{[12]}$

TGA was performed in Ar atmosphere with a heating rate of $10{ }^{\circ} \mathrm{C} \mathrm{min}-1$ from room temperature to $600{ }^{\circ} \mathrm{C}$ to investigate the thermal behavior of SGDY. As shown in Figure $4 \mathrm{a}, \mathrm{S}_{8}$ begins a rapid weight loss from $180{ }^{\circ} \mathrm{C}$ and completely losses weight at $320^{\circ} \mathrm{C}$, which is consistent with previous reports. ${ }^{[13]}$ In contrast, there is almost no weight loss below $320{ }^{\circ} \mathrm{C}$ for SGDY, indicating $\mathrm{S}_{8}$ does not exist. It is noted that the thermal stability of SGDY is improved compared with pristine GDY powder, which continuously loses weight until $600{ }^{\circ} \mathrm{C}$. Considering SGDY is synthesized by heating sublimed sulfur and GDY at $350{ }^{\circ} \mathrm{C}$, we investigate the structure evolution of GDY at $350^{\circ} \mathrm{C}$. Figure S1 (Supporting Information) displays the Raman spectrum of pure GDY after being heated at $350^{\circ} \mathrm{C}$, which shows similar peaks to GDY at room temperature, including a D band at $1356.6 \mathrm{~cm}^{-1}$, a $\mathrm{G}$ band at $1575.5 \mathrm{~cm}^{-1}$, and a peak of diacetylenic linkages $(-\mathrm{C} \equiv \mathrm{C}-\mathrm{C} \equiv \mathrm{C}-)$ at $2170.4 \mathrm{~cm}^{-1}$. In addition, the high-solution C 1s XPS spectra of GDY heated at $350{ }^{\circ} \mathrm{C}$ (Figure S2, Supporting Information) exhibit four main sub-peaks including $\mathrm{C}-\mathrm{C}\left(\mathrm{sp}^{2}\right), \mathrm{C} \equiv \mathrm{C}(\mathrm{sp}), \mathrm{C}-\mathrm{O}$, and $\mathrm{C}=\mathrm{O}$ bonds. Moreover, the area ratio of the $\mathrm{sp}$ and $\mathrm{sp}^{2}$-hybridized carbon atoms was obviously 2, suggesting GDY heated at $350{ }^{\circ} \mathrm{C}$ still features benzene rings linked through diacetylenic linkages. Based on the 

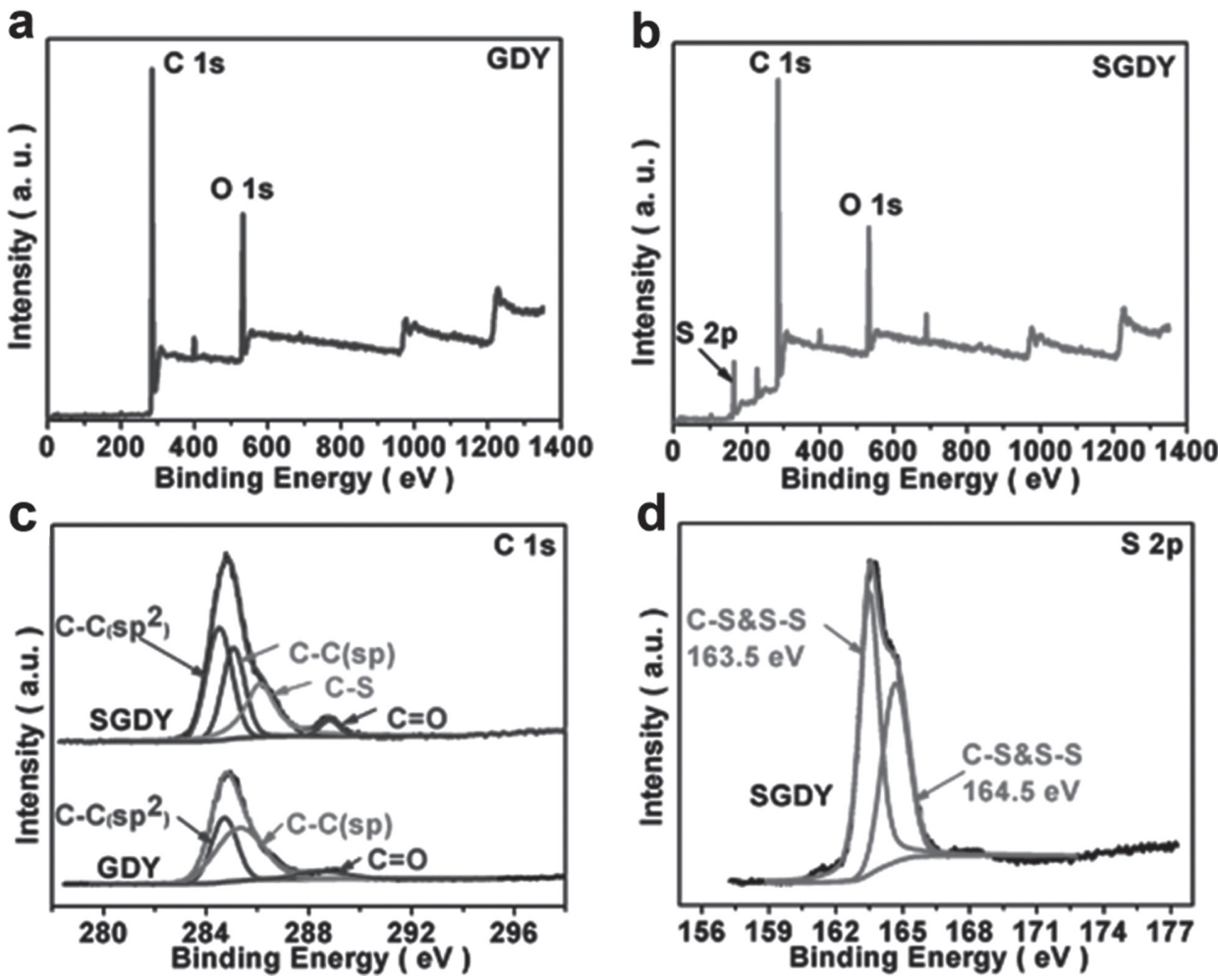

Figure 3. XPS survey spectra of a) GDY and b) SGDY. c) C 1s spectra of GDY and SGDY. d) S 2p spectra of SGDY.

above analysis, the structure evolution of GDY from room temperature to $350{ }^{\circ} \mathrm{C}$ can probably be assigned to volatilization of oligomers that are formed when synthesizing GDY via the cross-coupling reaction of hexaethynylbenzene in solution. The X-ray diffraction (XRD) patterns in Figure $4 \mathrm{~b}$ show broad peaks at $23^{\circ}$, suggesting the amorphous nature of the structures of pristine GDY and SGDY. The XRD pattern of SGDY does not contain any peaks of $\mathrm{S}_{8}$ crystalline (PDF\#53-1109), confirming efficient elimination of $\mathrm{S}_{8}$ by the $\mathrm{CS}_{2}$ treatment. According to the elemental analysis, SGDY contains $\approx 30.2 \mathrm{wt} \%$ sulfur.

Based on all the above discussion, it can be concluded that SGDY not only contains functional C-S species and $\mathrm{S}_{x}(2 \leq x \leq 4)$ species, serving as electrochemically reactive sites, but also composes of intrinsic electron/Li-ion conductive skeletons benefited from abundant conjugated linkages and triangle-like pores, respectively. So the proposed atomistic structure of SGDY is as shown in Figure 1a. The electrical conductivities of GDY and SGDY were also measured by the four-point probe method. The electrical conductivities of GDY and SGDY were tested to be $2.14 \times 10^{-4}$ and $4.37 \times 10^{-4} \mathrm{~S} \mathrm{~m}^{-1}$, respectively.

\subsection{Electrochemical Properties}

\subsubsection{Li-S Batteries}

It is well known that organic carbonate electrolytes such as ethylene carbonate (EC), dimethyl carbonate (DMC), or their

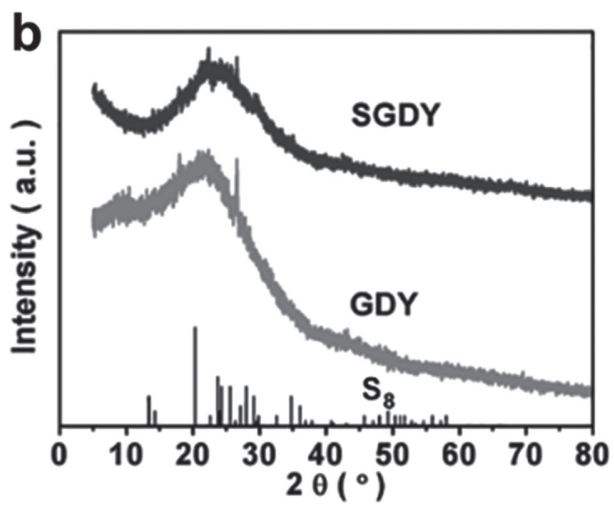

Figure 4. TGA curves of a) $S_{8}$, GDY, and SGDY. b) XRD patterns of $S_{8}$, GDY, and SGDY. 
mixtures are not suitable for the $\mathrm{S}_{8}$-based systems owing to their poor dissolubility of the intermediate high-order polysulfides. ${ }^{[31]}$ Moreover, the carbonate-based solvents were found to react with the nucleophilic sulfide anions. ${ }^{[32]}$ In our case, element $\mathrm{S}$ exists as short-sulfide mode such as $\mathrm{C}-\mathrm{S}$ bonds and short $\mathrm{S}-\mathrm{S}$ bonds instead of $\mathrm{S}_{8}$ in SGDY, so there would be no formation of high-order polysulfides, thus rending SGDY compatible with the carbonate-based electrolyte. To test the compatibility of our delicate-designed cathode, the electrochemical performance of SGDY was investigated in the potential range between 1.0 and $3.0 \mathrm{~V}$ versus $\mathrm{Li} / \mathrm{Li}^{+}$with $1 \mathrm{M} \mathrm{LiPF}_{6}$ dissolved in a mixture of EC and DMC (v:v = 1:1) as the electrolyte. Figure S3 (Supporting Information) shows the first chargedischarge curves of the SGDY cathode at a current density of $0.1 \mathrm{C}\left(1 \mathrm{C}=1672 \mathrm{~mA} \mathrm{~g}^{-1}\right)$. It is noted that there is only a single plateau at $1.6-1.7 \mathrm{~V}\left(\mathrm{vs} \mathrm{Li} / \mathrm{Li}^{+}\right)$in the first discharge and one plateau at $2.2 \mathrm{~V}\left(\mathrm{vs} \mathrm{Li} / \mathrm{Li}^{+}\right)$in the first charge rather than the two-step reaction of conventional $\mathrm{Li}-\mathrm{S}$ batteries, indicating complete elimination of the formation of high-order polysulfide intermediates, thus avoiding the shuttle effect, which is beneficial for the improvement of cyclability. SGDY exhibits a first cycle reversible specific capacity as high as $960.9 \mathrm{mAh} \mathrm{g}^{-1}$ (based on sulfur mass). After the first cycle with an irreversible capacity, the following cycles (shown in Figure 5a) are overlapped with high reversibility and $\approx 100 \%$ Coulombic efficiency. Furthermore, the lithiation voltage in the first cycle is lower than that in the following cycles, indicative of reduced overpotential, which is probably due to the introduction of large defects in the first lithiation. ${ }^{[30]}$ The large irreversible capacity loss in the first cycle is mainly caused by the catalytic reduction of electrolyte solvents on the fresh surface of the SGDY cathode.

To reveal the electrochemical reaction mechanism of SGDY cathode, cyclic voltammetry $(\mathrm{CV})$ test was performed in the potential range of $1.0-3.0 \mathrm{~V}$ versus $\mathrm{Li} / \mathrm{Li}^{+}$at a scanning rate of $0.1 \mathrm{mV} \mathrm{s}^{-1}$. CV curves (Figure 5b) show only one pair of reversible redox peaks, confirming a different mechanism from $\mathrm{S}_{8}$-Li multiple-step reaction. ${ }^{[33]}$ Overpotential exists due to a solid-tosolid phase transition. Higher current response and lower lithiation potential in the first discharge than those in the following cycles are observed, which are consistent with the dischargecharge profiles. Figure $5 c$ displays the rate capability of the SGDY cathode. At a high rate of $2 \mathrm{C}$ (i.e., $3344 \mathrm{~mA} \mathrm{~g}^{-1}$ ), SGDY still exhibits a high specific capacity of $503.1 \mathrm{mAh} \mathrm{g}^{-1}$. When the current density is reset to $0.1 \mathrm{C}$, the capacity reverses back to $949.2 \mathrm{mAh} \mathrm{g}^{-1}$, implying an outstanding rate performance. The outstanding rate capability can be attributed to the unique structure of SGDY, which possesses a conductive carbon skeleton with high Li-ion mobility. ${ }^{[14]}$ The SGDY cathode also shows superior cycle stability as shown in Figure 5d, with reversible capacities of 821.4 and $713.7 \mathrm{mAh} \mathrm{g}^{-1}$ after 100 cycles at current densities of 0.5 and $1 \mathrm{C}$, respectively. Besides, the Coulombic efficiency is nearly $100 \%$ in every cycle. That the SGDY cathode shows excellent electrochemical performance in carbonate-based electrolytes, which are more resistant to high temperature than glyme-based and dioxolane electrolytes, would putting forward the development of safer Li-S batteries.

Table S1 (Supporting Information) compares the cycle performance of different sulfur cathodes. It can be seen that our SGDY cathode shows comparable cycle performance to that of reported
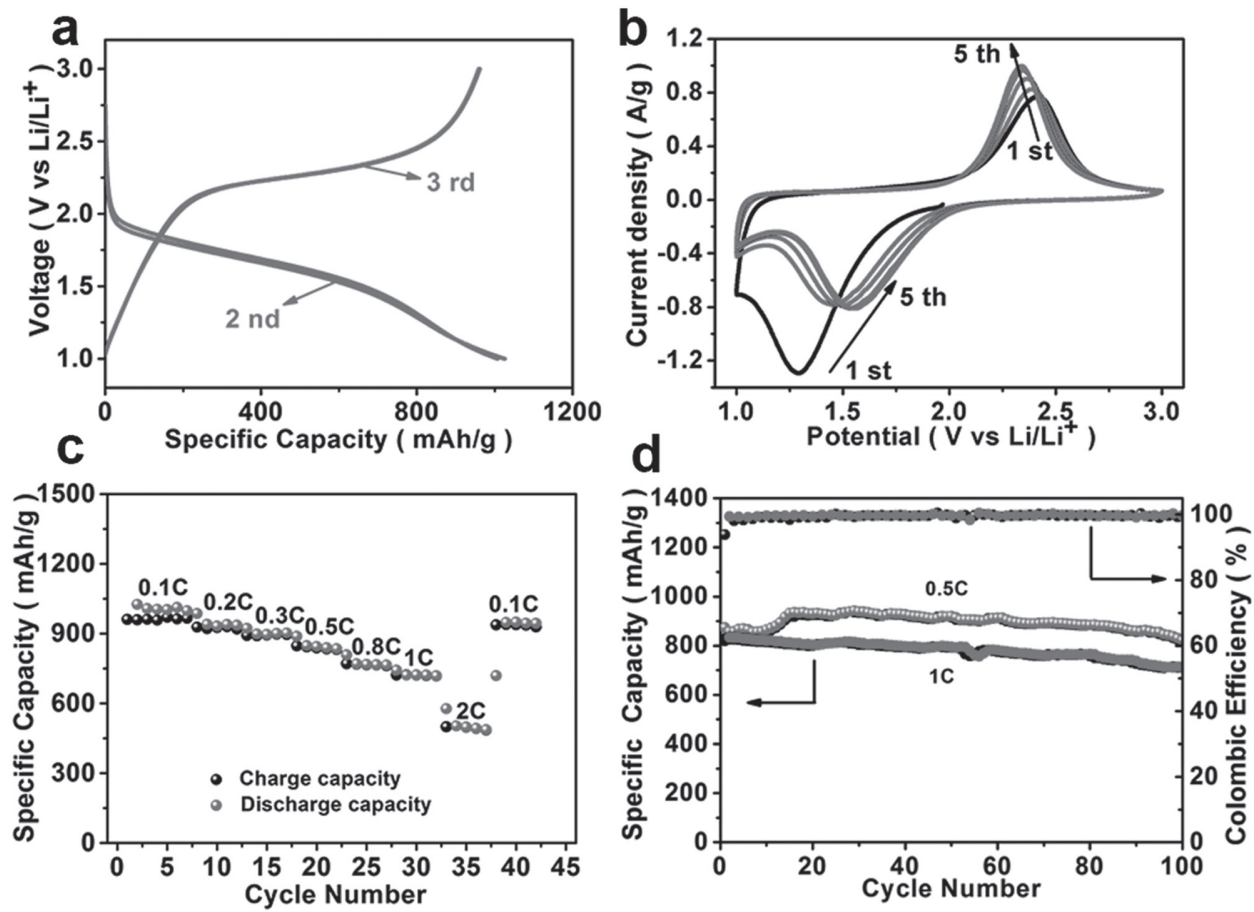

Figure 5. a) The second and third charge-discharge profiles of the SGDY cathode at a current density of $0.1 \mathrm{C}\left(1 \mathrm{C}=1672 \mathrm{~mA} \mathrm{~g}{ }^{-1}\right)$. b) CV curves of the SGDY cathode at a scan rate of $0.1 \mathrm{mV} \mathrm{s}^{-1}$. c) Rate performance of the SGDY cathode. d) Cycling performance of the SGDY cathode at current densities of 0.5 and $1 \mathrm{C}$, respectively. 
sulfur cathodes. ${ }^{[11,12,20,34-41]}$ Meantime, this is the first exploration of graphdiyne (GDY) for Li-S batteries. It is also noted that GDY is synthetically approachable via the cross-coupling reaction of hexaethynylbenzene (monomer). It may be possible to synthesize GDY with controlled structure by modifying reaction conditions or GDY analogs by employing different monomers. In fact, Liu and co-workers synthesized graphdiyne nanowalls using a modified Glaser-Hay coupling reaction. ${ }^{[42]}$ The electrochemical performance of SGDY cathode could be further improved by tuning the structure and morphology of GDY. Our delicately designed strategy could open a new avenue for preparing sulfur cathode from molecular design.

Electrochemical impedance spectroscopy (EIS) was carried out to understand the interfacial charge transfer and Li-ion diffusion process in the SGDY electrode during varied cycles. The Nyquist plots are fitted with the equivalent circuit inset in Figure 6a. In the equivalent circuit, $R_{\mathrm{S}}$ represents the combination resistance including the intrinsic resistance of the electrodes, the contact resistance between the electrodes and current collectors, and the ionic resistance of the electrolyte; $R_{\mathrm{SEI}}$ and CPE1 are the resistance and capacitance of the solid electrolyte interface (SEI) formed on the electrode respectively; $R_{\mathrm{CT}}$ and CPE2 represent the charge-transfer resistance and the double-layer capacitance, respectively; and $W_{\mathrm{O}}$ is the Warburg impedance related to the diffusion of Li-ions into the bulk electrode. Table S2 (Supporting Information) lists all the fitting parameters of the above resistance values. $R_{\mathrm{s}}$ changes slightly through the 100 cycles (Figure 6b), which further confirms no presence of high-order polysulfide intermediates, thus shuttle effect was avoided essentially. ${ }^{[43]}$ The value of $R_{\mathrm{CT}}$ gradually decreases from $54.94 \Omega$ to $12.1 \Omega$, which may be due to the improved conducting property and explaining the reduced overpotential mentioned above.

The typical scanning electron microscopy (SEM) images of the cathode before and after cycling are also provided in Figure S4 (Supporting Information). Before cycling, SGDY mixes with Super P uniformly (Figure S4a, Supporting Information). After cycling, the film-like-deposited materials are observed around the SGDY and Super P particles in Figure S4b (Supporting Information), which might be the SEI layer formed by the electrolyte solvents reduction as discussed before.

\subsection{2. $M g-S$ Batteries}

The commonly used Grignard reagent based electrolytes, e.g., APC electrolytes, are nucleophilic and can react with an electrophilic $\mathrm{S}_{8}$ cathode. ${ }^{[2]}$ Figure S5 (Supporting Information) shows the discharge-charge curves of $\mathrm{S}_{8}$-CNT cathode using APC electrolytes with extra addition of LiCl. It can be obviously seen that the cathode overcharges, indicating $\mathrm{S}_{8}$-based cathodes are not compatible with APC electrolytes. For SGDY, the electrophilicity of sulfur is decreased by forming short-sulfide units, making SGDY compatible with APC electrolytes. The electrochemical performance of SGDY cathode was investigated within a potential range between 0.4 and $2.1 \mathrm{~V}$ versus $\mathrm{Mg} / \mathrm{Mg}^{2+}$ using APC electrolyte with $\mathrm{LiCl}$ additive. Figure $7 \mathrm{a}$ shows the initial discharge/charge curves of the SGDY cathode. The SGDY cathode delivers a large initial discharge capacity of $1124.9 \mathrm{mAh} \mathrm{g}^{-1}$ and a charge capacity of $539.3 \mathrm{mAh} \mathrm{g}^{-1}$. Except for the first cycle, the following cycles (shown in Figure $7 \mathrm{~b}$ ) are overlapped, indicating a high reversibility. Figure 7c displays the rate performance of SGDY. It is shown that the capacity decreases slightly with the increase of the current density, revealing a superior rate capability. The discharge capacity of the 36th cycle is $458.9 \mathrm{mAh} \mathrm{g}^{-1}$, corresponding to $77.3 \%$ of capacity retention (Figure 7e). In view of cycle life and capacity, our Mg-S batteries are competitive compared with previous reports (Figure 7d). ${ }^{[2,3,44-46]}$ Figure 8 shows the following discharge-charge curves of the SGDY cathode from the 36th cycle. It can be seen that the irreversible capacity gets larger and larger when cycled for 41 cycles, which may be due to the reduction of electrolytes or the corrosion of coin cell assemblies. Both of them would lead to performance deterioration. ${ }^{[47,48]}$ In spite of this, the cycle performance of our $\mathrm{Mg}-\mathrm{S}$ batteries is superior to previously published reports as shown in Table S3 (Supporting Information). Table S3 (Supporting Information) lists the electrochemical performance of different types of $\mathrm{Mg}-\mathrm{S}$ batteries in detail. It is found that the SGDY cathode shows better rate capability and cycle stability. It is also noted that this is the first implementation case of a reliable $\mathrm{Mg}-\mathrm{S}$ system based on nucleophilic APC electrolytes.

Figure S6 (Supporting Information) displays the electrochemical performance of the SGDY cathode using APC
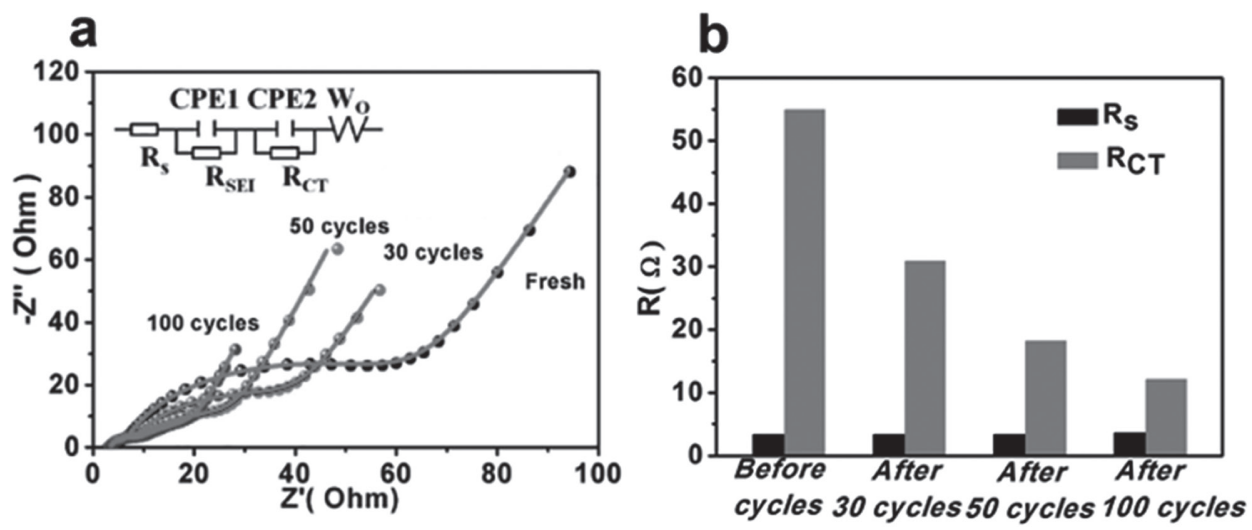

Figure 6. a) EIS spectra of the SGDY cathode during varied cycles at a current density of $1 \mathrm{C}$ and the equivalent circuit. b) Kinetic parameters of the SGDY cathode during varied cycles. 

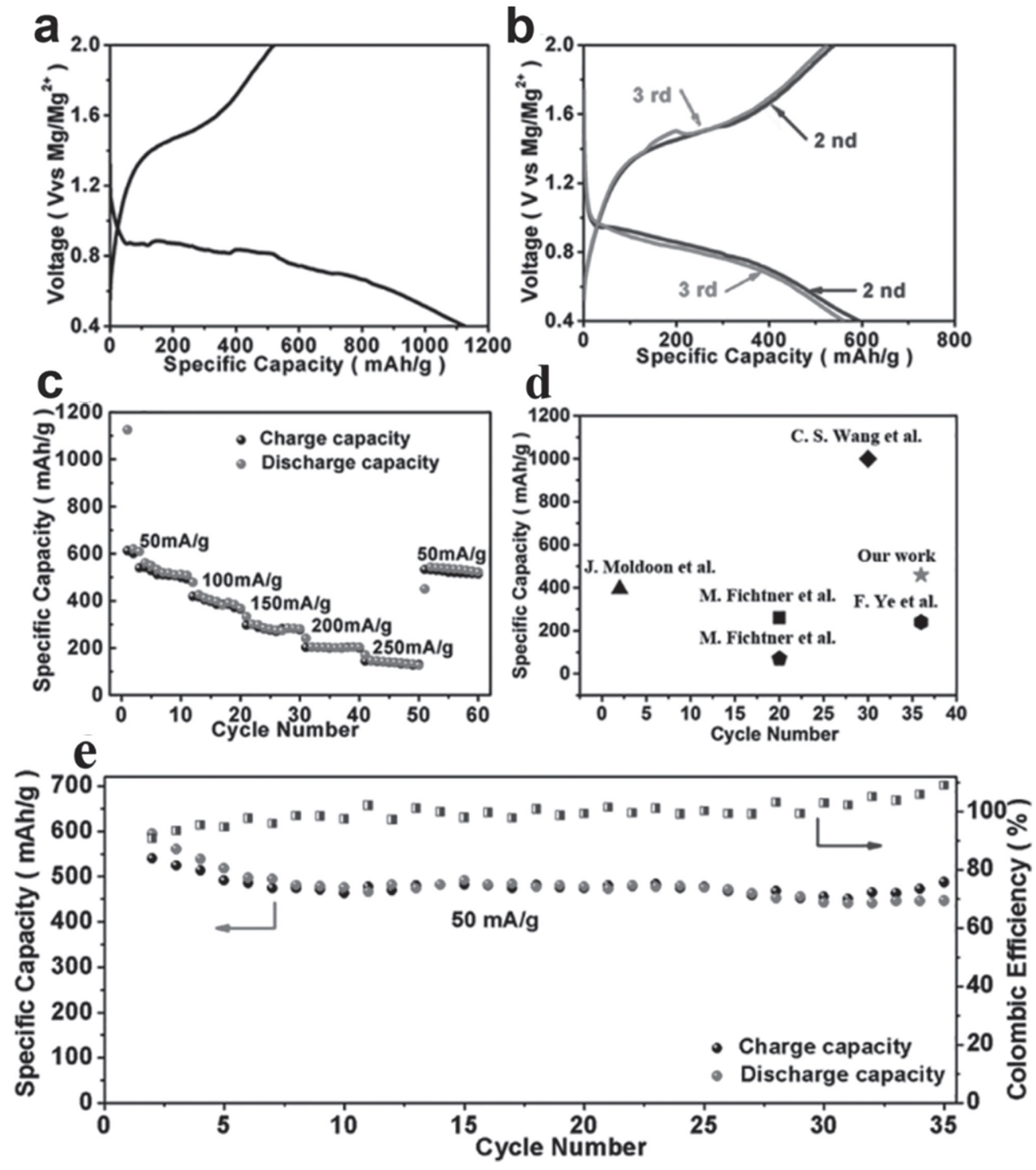

Figure 7. a) The initial charge-discharge curves of the SGDY cathode using APC electrolyte with LiCl additive at a current density of $50 \mathrm{~mA} \mathrm{~g} \mathrm{~g}^{-1}$. b) The second and third charge-discharge profiles of the SGDY cathode at a current density of $50 \mathrm{~mA} \mathrm{~g}^{-1}$. c) Rate performance of SGDY cathode. d) Cycle life and capacity comparison between our Mg-S batteries with previous studies. e) Cycling performance of the SGDY cathode at a current density of $50 \mathrm{~mA} \mathrm{~g}{ }^{-1}$.

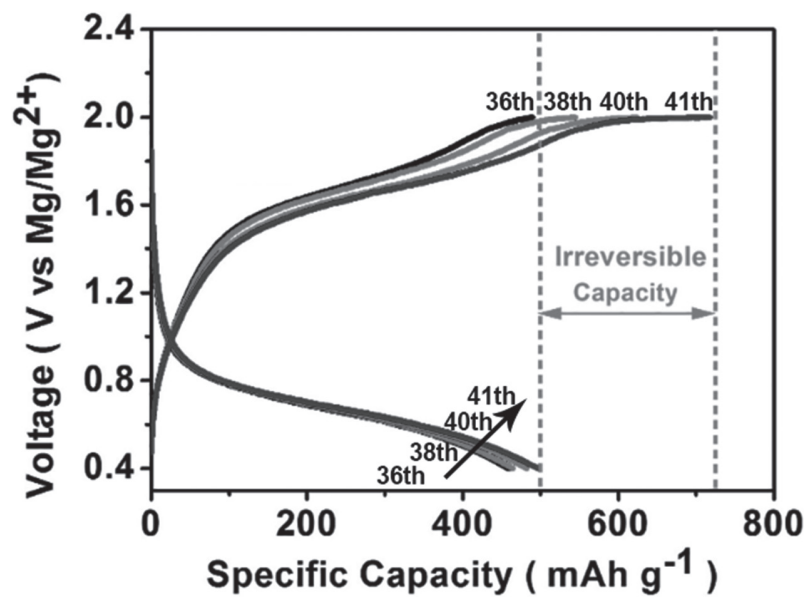

Figure 8. Different cycles of discharge-charge curves of the SGDY cathode. electrolyte without $\mathrm{LiCl}$ additive. The SGDY cathode shows almost no capacity without $\mathrm{LiCl}$ additive. The phenomenon is similar to a previous report in which rapid capacity drop was observed for sulfur cathode in Mg-only electrolyte, while the reversibility is extraordinarily improved with the presence of $\mathrm{Li}^{+} .{ }^{[46]}$ The explanation of such interesting result was proposed as that $\mathrm{Li}^{+}$could activate the inactive $\mathrm{MgS}$ and $\mathrm{MgS}_{2}$ by transforming them into rechargeable $\mathrm{Li}_{2} \mathrm{~S}$ and $\mathrm{Li}_{2} \mathrm{~S}_{2}$.

\section{Conclusion}

In summary, we have successfully synthesized a novel SGDY cathode explored for $\mathrm{Li}-\mathrm{S}$ and $\mathrm{Mg}-\mathrm{S}$ batteries, both of which show excellent electrochemical performances including large capacity, superior rate capability, stable capacity retention, and high Coulombic efficiency except for the first cycle. Meanwhile, 
the SGDY cathode is compatible with commercially available carbonate-based electrolyte for $\mathrm{Li}-\mathrm{S}$ batteries and the commonly used Grignard reagent-based electrolytes (APC electrolytes) for $\mathrm{Mg}-\mathrm{S}$ batteries. What's more, the assembled $\mathrm{Mg}-\mathrm{S}$ batteries showed high and stable capacity up to 36 cycles, which is superior to previous reports. Due to the richness and flexibility of the graphyne family, it is promising to increase the content of sulfur by tuning graphyne structure, thus offering a huge potential toward on low-cost and high-performance sulfur-based batteries.

\section{Experimental section}

Material Synthesis: For SGDY, first, GDY powder and sublimed sulfur were thoroughly mixed with a mass ratio of $m_{\mathrm{s}}: m_{\mathrm{CDY}}=50: 50$ in a quartz mortar for $1 \mathrm{~h}$ to yield a black mixture. Then the black mixture was heated at $350{ }^{\circ} \mathrm{C}$ for $3 \mathrm{~h}$ in a tube furnace with $\mathrm{Ar}$ atmosphere. At this temperature, the sublimed sulfur $\left(S_{8}\right)$ was dissociated into short chain sulfur $\left(S_{x}, 1 \leq x \leq 4\right)$, some of which chemically linked to carbon-carbon triple bonds and some diffused into the triangular pores and interlayer spacing of GDY. The obtained sample was treated with $\mathrm{CS}_{2}$ for no less than three times to remove the excess $S_{8}$ and then dried in a vacuum oven at $100{ }^{\circ} \mathrm{C}$ for $2 \mathrm{~h}$, noted as SGDY. The $\mathrm{S}_{8}$-CNTs composite as control compound was prepared by a simple melting-infusion method. Specifically, sublimed sulfur $\left(S_{8}\right)$ and multiwalled carbon nanotubes (CNTs) were thoroughly mixed with a mass ratio of $m_{\mathrm{s}}: m_{\mathrm{CNTs}}=60: 40$ by ball-milling and then heated at $155^{\circ} \mathrm{C}$ for $24 \mathrm{~h}$ to obtain $\mathrm{S}_{8}$-CNTs composite.

Samples Characterization: The morphologies of the materials were characterized by field emission SEM (HITACHI S-4800) equipped with an energy dispersive X-ray spectrometry. TEM and HRTEM images were obtained using TEM (JEOL 2011F and 2100F). Raman spectra were recorded at the room temperature using a Thermo Scientific DXRxi system with a $532 \mathrm{~nm}$ laser. FTIR was recorded on a Bruker VECTOR 22 spectrometer in the frequency range of $4000-500 \mathrm{~cm}^{-1}$. XRD patterns were recorded on X-ray powder diffractometer (D8 ADVANCE) with $\mathrm{Cu}$ $K \alpha$ radiation. TCA was performed in $\mathrm{Ar}$ atmosphere with a heating rate of $10{ }^{\circ} \mathrm{C} \mathrm{min}-1$ from room temperature to $600{ }^{\circ} \mathrm{C}$. XPS analysis was performed by using VG Scientific ESCALab220i-XL X-ray photoelectron spectrometer with $\mathrm{K} \alpha$ radiation as the excitation sources. Elemental analysis of $\mathrm{C}, \mathrm{N}, \mathrm{S}$, and $\mathrm{H}$ was conducted using a LECO TruSpec Micro elemental analyzer. The electrical conductivities of GDY and SGDY were measured by the four-point probe method using an RTS-9 Digital Instrument.

Electrochemical Evaluation: For Li-S batteries, the SGDY electrode slurry consisted of 70\% SGDY, 20\% Super P, and 10\% binder (sodium carboxymethyl cellulose and styrene butadiene rubber dissolved in water). The cathode was prepared by casting the slurry onto aluminum foil using a doctor blade and dried at $60^{\circ} \mathrm{C}$ for $12 \mathrm{~h}$ in a vacuum oven. The 2032 coin cells were assembled in an Ar-filled glove box to measure the electrochemical performance of the as-prepared cathode with lithium metal as the counter electrode and reference electrode, the glass-fiber film as separator, and $1 \mathrm{M} \mathrm{LiPF}_{6}$ dissolved in a mixture of EC and DMC $(\mathrm{v}: \mathrm{v}=1: 1)$ as the electrolyte. $50 \mu \mathrm{L}$ of electrolyte $\left(1 \mathrm{M} \mathrm{LiPF}_{6}\right.$ dissolved in a mixture of EC and DMC) was used in a single coin cell. Galvanostatic charge/discharge tests were performed in a potential range between 1.0 and $3.0 \mathrm{~V}$ versus $\mathrm{Li} / \mathrm{Li}^{+}$under a LAND-CT2001 instrument at room temperature. The specific capacity was calculated on the basis of the active sulfur obtained from elemental analysis and the mass loading of sulfur for each electrode was $1 \mathrm{mg} \mathrm{cm} \mathrm{cm}^{-2}$. CV was performed using an electrochemical workstation $(\mathrm{CHI} 660 \mathrm{C})$ at a scanning rate of $0.1 \mathrm{mV} \mathrm{s}^{-1}$. EIS was performed by using an IM6e electrochemical workstation in the frequency range of $1 \mathrm{MHz}-100 \mathrm{mHz}$.

The preparation of the cathode for $\mathrm{Mg}-\mathrm{S}$ batteries is as follows: with a weight ratio of 70: 20:10, the SCDY (or S8-CNTs) powder, polytetrafluoroethylene, and Super $\mathrm{P}$ were mixed together in a mortar. The mixture were rolled into thin sheets and then cut into electrodes with square shape. The APC electrolyte was synthesized according to previous reports. ${ }^{[49]}$ In a typical procedure, $0.6666 \mathrm{~g}$ anhydrous $\mathrm{AlCl}_{3}$ was dissolved into $5 \mathrm{~mL}$ purified tetrahydrofuran (THF) solvent with vigorous magnetic stirring for at least $1 \mathrm{~h}$. Then, $5 \mathrm{~mL}$ of $2 \mathrm{~m}$ phenyl magnesium chloride in THF was carefully dropped into the above solution followed by vigorous magnetic stirring for $1 \mathrm{~d}$. Anhydrous $0.021 \mathrm{~g} \mathrm{LiCl}$ was added into the as-prepared solution to obtain Mg-based electrolyte. The 2032 coin cells were assembled in an Ar-filled glove box with magnesium metal as the counter electrode and reference electrode, the glassfiber film as separator, and the Mg-based electrolyte as the electrolyte. $80 \mu \mathrm{L}$ of the Mg-based electrolyte was used in a single coin cell. The charge-discharge properties of the SGDY electrodes were studied by galvanostatic discharge-charge experiments within a potential between 0.4 and $2.1 \mathrm{~V}$ versus $\mathrm{Mg} / \mathrm{Mg}^{2+}$. The specific capacity was calculated on the basis of the active sulfur obtained from elemental analysis and the mass loading of sulfur was $1 \mathrm{mg} \mathrm{cm}$.

\section{Supporting Information}

Supporting Information is available from the Wiley Online Library or from the author.

\section{Acknowledgements}

This work was supported by Qingdao Key Lab of Solar Energy Utilization and Energy Storage Technology, the National Natural Science Foundation of China program (Grant No. 21271180), and the Key Technology Research Projects of Qingdao (Grant No.13-CX-10).

\section{Conflict of Interest}

The authors declare no conflict of interest.

\section{Keywords}

high $\mathrm{Li}^{+}$mobility, metal-sulfur batteries, short sulfide unites, shuttle effect, sulfide graphdiyne

Received: July 4, 2017

Revised: August 8, 2017

Published online: September 28, 2017

[1] X. L. Ji, K. T. Lee, L. F. Nazar, Nat. Mater. 2009, 8, 500.

[2] H. S. Kim, T. S. Arthur, G. D. Allred, J. Zajicek, J. G. Newman, A. E. Rodnyansky, A. G. Oliver, W. C. Boggess, J. Muldoon, Nat. Commun. 2011, 2, 427.

[3] W. Li, S. Cheng, J. Wang, Y. Qiu, Z. Zheng, H. Lin, S. Nanda, Q. Ma, Y. Xu, F. Ye, Angew. Chem. Int. Ed. 2016, 128, 6516.

[4] A. Manthiram, Y. Fu, Y. S. Su, Acc. Chem. Res. 2012, 46, 1125.

[5] M. Shaibani, A. Akbari, P. Sheath, C. D. Easton, P. C. Banerjee, K. Konstas, A. Fakhfouri, M. Barghamadi, M. M. Musameh, A. S. Best, ACS Nano 2016, 10, 7768.

[6] Z. Zhang, Z. Li, F. Hao, X. Wang, Q. Li, Y. Qi, R. Fan, L. Yin, Adv. Funct. Mater. 2014, 24, 2500

[7] X. Ji, L. F. Nazar, J. Mater. Chem. A 2010, 20, 9821.

[8] R. J. Chen, T. Zhao, F. Wu, Chem. Commun. 2015, 51, 18. 
[9] L. Ji, M. Rao, H. Zheng, L. Zhang, Y. Li, W. Duan, J. Guo, E. J. Cairns, Y. Zhang, J. Am. Chem. Soc. 2011, 133, 18522.

[10] G. Zhou, S. Pei, L. Li, D. W. Wang, S. Wang, K. Huang, L. C. Yin, F. Li, H. M. Cheng, Adv. Mater. 2014, 26, 625.

[11] M. Q. Zhao, X. F. Liu, Q. Zhang, G. L. Tian, J. Q. Huang, W. C. Zhu, F. Wei, ACS Nano 2012, 6, 10759.

[12] S. Xin, L. Gu, N. H. Zhao, Y. X. Yin, L. J. Zhou, Y. G. Guo, L. J. Wan, J. Am. Chem. Soc. 2012, 134, 18510.

[13] S. Zheng, Y. Wen, Y. Zhu, Z. Han, J. Wang, J. Yang, C. Wang, Adv. Energy Mater. 2014, 4, 1400482.

[14] H. Zhang, M. Zhao, X. He, Z. Wang, X. Zhang, X. Liu, J. Phys. Chem. C 2011, 115, 8845.

[15] C. Huang, S. Zhang, H. Liu, Y. Li, G. Cui, Y. Li, Nano Energy 2015, $11,481$.

[16] S. Zhang, H. Liu, C. Huang, G. Cui, Y. Li, Chem. Commun. 2015, 51, 1834.

[17] H. P. Du, H. Yang, C. S. Huang, J. J. He, H. B. Liu, Y. L. Li, Nano Energy 2016, 22, 615.

[18] H. Y. Zhang, X. J. He, M. W. Zhao, M. Zhang, L. X. Zhao, X. J. Feng, Y. H. Luo, J. Phys. Chem. C 2012, 116, 13364.

[19] J. Y. Zhou, X. Gao, R. Liu, Z. Q. Xie, J. Yang, S. Q. Zhang, G. M. Zhang, H. B. Liu, Y. L. Li, J. Zhang, Z. F. Zhong, J. Am. Chem. Soc. 2015, 137, 7596

[20] B. Duan, W. Wang, A. Wang, K. Yuan, Z. Yu, H. Zhao, J. Qiu, Y. Yang, J. Mater. Chem. A 2013, 1, 13261.

[21] P. T. Dirlam, A. G. Simmonds, T. S. Kleine, N. A. Nguyen, L. E. Anderson, A. O. Klever, A. Florian, P. J. Costanzo, P. Theato, M. E. Mackay, RSC Adv. 2015, 5, 24718.

[22] Z. Ni, Y. Wang, T. Yu, Z. Shen, Nano Res. 2008, 1, 273.

[23] J. Wang, S. Zhang, J. Zhou, R. Liu, R. Du, H. Xu, Z. Liu, J. Zhang, Z. Liu, Phys. Chem. Chem. Phys. 2014, 16, 11303.

[24] J. S. Kim, T. H. Hwang, B. G. Kim, J. Min, J. W. Choi, Adv. Funct. Mater. 2014, 24, 5359.

[25] G. X. Li, Y. L. Li, H. B. Liu, Y. B. Guo, Y. J. Li, D. B. Zhu, Chem. Commun. 2010, 46, 3256.

[26] X.-G. Yu, J.-Y. Xie, J. Yang, H.-J. Huang, K. Wang, Z.-S. Wen, J. Electroanal. Chem. 2004, 573, 121.

[27] G. Li, J. Sun, W. Hou, S. Jiang, Y. Huang, J. Geng, Nat. Commun. 2016, 7, 10601.

[28] M. Q. Zhao, M. Sedran, Z. Ling, M. R. Lukatskaya, O. Mashtalir, M. Ghidiu, B. Dyatkin, D. J. Tallman, T. Djenizian, M. W. Barsoum, Angew. Chem. Int. Ed. 2015, 54, 4810.
[29] S. Zheng, P. Han, Z. Han, H. Zhang, Z. Tang, J. Yang, Sci. Rep. 2014, 4, 4842.

[30] L. Ma, H. L. Zhuang, S. Wei, K. E. Hendrickson, M. S. Kim, G. Cohn, R. G. Hennig, L. A. Archer, ACS Nano 2015, 10, 1050

[31] R. Rauh, F. Shuker, J. Marston, S. Brummer, J. Inorg. Nucl. Chem. 1977, 39, 1761.

[32] Z. Li, L. Yuan, Z. Yi, Y. Sun, Y. Liu, Y. Jiang, Y. Shen, Y. Xin, Z. Zhang, Y. Huang, Adv. Energy Mater. 2014, 4, 1301473.

[33] A. Manthiram, Y. Fu, S. H. Chung, C. Zu, Y. S. Su, Chem. Rev. 2014, $114,11751$.

[34] G. Zhou, L.-C. Yin, D.-W. Wang, L. Li, S. Pei, I. R. Gentle, F. Li, H.-M. Cheng, ACS Nano 2013, 7, 5367.

[35] Y.-X. Wang, L. Huang, L.-C. Sun, S.-Y. Xie, G.-L. Xu, S.-R. Chen, Y.-F. Xu, J.-T. Li, S.-L. Chou, S.-X. Dou, J. Mater. Chem. 2012, 22, 4744.

[36] R. Chen, T. Zhao, J. Lu, F. Wu, L. Li, J. Chen, G. Tan, Y. Ye, K. Amine, Nano Lett. 2013, 13, 4642.

[37] F. Sun, J. Wang, H. Chen, W. Li, W. Qiao, D. Long, L. Ling, ACS Appl. Mater. Interfaces 2013, 5, 5630.

[38] K. Zhang, Q. Zhao, Z. Tao, J. Chen, Nano Res. 2013, 6, 38.

[39] J. L. Shi, C. Tang, H. J. Peng, L. Zhu, X. B. Cheng, J. Q. Huang, W. Zhu, Q. Zhang, Small 2015, 11, 5243.

[40] J. Wang, J. Yang, J. Xie, N. Xu, Adv. Mater. 2002, 14, 963.

[41] Y. Xu, Y. Wen, Y. Zhu, K. Gaskell, K. A. Cychosz, B. Eichhorn, K. Xu, C. Wang, Adv. Funct. Mater. 2015, 25, 4312.

[42] J. Zhou, X. Gao, R. Liu, Z. Xie, J. Yang, S. Zhang, G. Zhang, H. Liu, Y. Li, J. Zhang, J. Am. Chem. Soc. 2015, 137, 7596.

[43] F. Chen, J. Yang, T. Bai, B. Long, X. Y. Zhou, Electrochim. Acta 2016, 192, 99.

[44] Z. Zhao, X. Y. Zhao, D. Wang, T. Diemant, R. J. Behm, M. Fichtner, Adv. Energy Mater. 2015, 5, 1401155.

[45] B. P. Vinayan, Z. R. Zhao, T. Diemant, V. S. K. Chakravadhanula, N. I. Schwarzburger, M. A. Cambaz, R. J. Behm, C. Kübel, M. Fichtner, Nanoscale 2016, 8, 3296.

[46] T. Gao, M. Noked, A. J. Pearse, E. Gillette, X. L. Fan, Y. J. Zhu C. Luo, L. M. Suo, M. A. Schroeder, K. Xu, S. B. Lee, G. W. Rubloff, C. S. Wang, J. Am. Chem. Soc. 2015, 137, 12388.

[47] S. Yagi, A. Tanaka, Y. Ichikawa, T. Ichitsubo, E. Matsubara, J. Electrochem. Soc. 2013, 160, C83.

[48] D. Lv, T. Xu, P. Saha, M. K. Datta, M. L. Gordin, A. Manivannan P. N. Kumta, D. Wang, J. Electrochem. Soc. 2013, 160, A351.

[49] Y. Cheng, Y. Shao, J. Zhang, V. L. Sprenkle, J. Liu, G. Li, Chem. Commun. 2014, 50, 9644. 